\title{
Active Suppression of Narrowband Noise by Multiple Secondary Sources
}

\author{
Seokhoon Ryu, Yun Jung Park, and Young-Sup Lee \\ Department of Embedded Systems Engineering, University of Incheon, 119 Academy-ro, Yeonsu-gu, \\ Incheon 406-772, Republic of Korea
}

Correspondence should be addressed to Young-Sup Lee; ysl@incheon.ac.kr

Received 19 March 2015; Revised 23 June 2015; Accepted 28 June 2015

Academic Editor: Marco Anisetti

Copyright (C) 2016 Seokhoon Ryu et al. This is an open access article distributed under the Creative Commons Attribution License, which permits unrestricted use, distribution, and reproduction in any medium, provided the original work is properly cited.

This study presents theoretical and experimental investigation on the active suppression of narrowband noise with $\mathrm{C} 1, \mathrm{C} 1.5$, and $\mathrm{C} 2$ components by using multichannel secondary sources in a duct. The quality manipulation in the duct was controlled by changing quality factors which were incorporated into a multichannel FxLMS algorithm. The algorithm is extensively investigated in both theory and real-time control experiment. After analysing the primary and secondary paths of the duct system, an acoustic narrowband signal was chosen as a primary noise and the impulse responses were implemented as the secondary path models. Control results show that the quality factors in the algorithm that was implemented in a dSPACE 1104 provide a stable and excellent response compared to before control. It is obvious that the lower quality factor cancels the more primary noise as defined in the theory although the attenuation levels are not exactly and inversely proportional to the quality factor. The results in this study can be used for practical active sound quality control systems.

\section{Introduction}

The active noise control (ANC) systems seek usually to maximize the attenuation of a primary noise by cancelling the unwanted noise based on the principle of superposition $[1,2]$. It takes advantage of the biologically inspired adaptive feedforward learning algorithms such as the filteredreference least mean square (FxLMS) to compensate for the effect of the secondary path in order to ensure convergence $[1,2]$. This ANC has been widely applied successfully to many applications such as airplanes, cars, headsets, mobile devices, and other consumer electronics.

However, in contrast to an ANC system, in some applications it is necessary to retain residual noise with a specified target profile over frequency $[3,4]$ because some intentional residual noise could provide better natural feeling rather than simply minimizing the residual noise. This approach is known as an active sound quality control (ASQC) and it can be implemented by incorporating some factors in its algorithm. A typical ASQC system, therefore, needs to have a capability of attenuating unwanted noise to a certain level and, at the same time, of enhancing wanted noise to a predefined target level. An adaptive noise equalization (ANE) is one of the concepts and has been extending to control narrowband and broadband noises [5-8]. Although a narrowband ANC system can reduce narrowband noise components at maximum, a narrowband ANE system can be independently manipulated.

The duct systems are widely applied to a number of different buildings, factories, and facilities. In some applications, such as clean rooms for precise manufacturing, they need to keep a comfortable noise level for workers and this leads to product quality. Also excessive reduction of noise can cause uncomfortable awkward silence. Thus the quality factor allows operating the noise reduction level with the ANC systems.

In this study, thus, an active sound quality control (ASQC) system using this ANE algorithm based the FxLMS is investigated in depth in order to control narrowband noise, which are dominant in a duct, separately or altogether in terms of sound quality control. For the selective quality control, a quality factor or parameter which is predetermined is applied in the real-time FxLMS adaptive algorithm. 


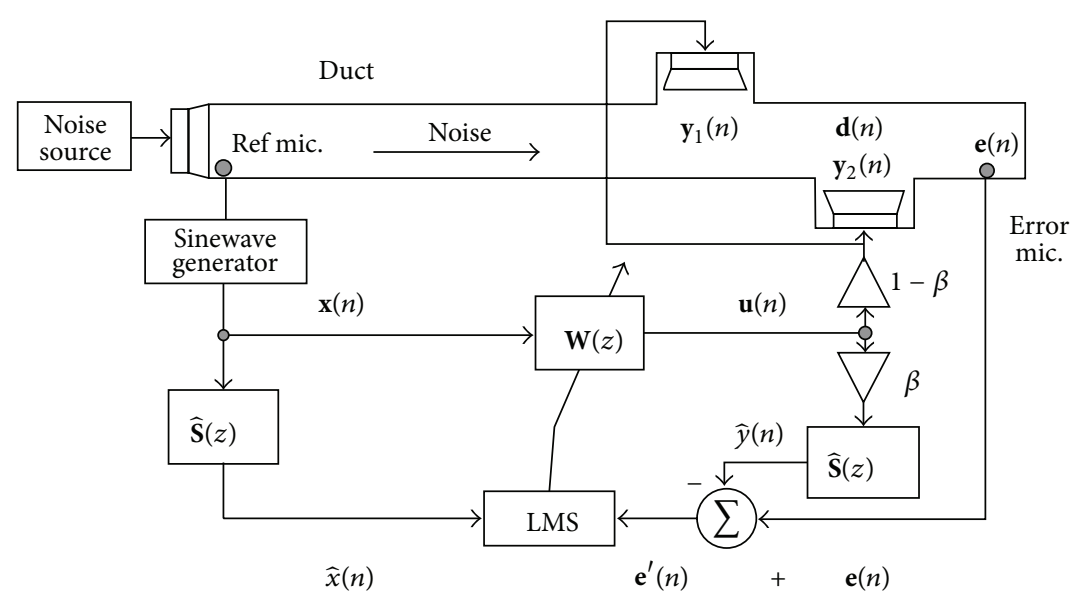

FIGURE 1: Active sound quality control for narrowband noise using a factor $\beta$ in a duct.

The rest of the paper is organized as follows. In Section 2, theoretical considerations are described to derive proper equations for sound quality control based on the multichannel FxLMS algorithm with a quality factor matrix in a duct. Section 3 presents the experimental arrangement including the test duct and control board for the real-time control. In the same section, the primary and secondary paths are discussed and the modeling of the secondary path to implement in the control algorithm is included. Intensive analysis and discussions from the measured results from the real-time sound quality control in terms of time and frequency domain are written in Section 4. Finally, conclusions are summarized in Section 5.

\section{Theoretical Considerations}

2.1. Multichannel Active Sound Quality Control for Narrowband Noise. An ANC system with an adaptive feedforward approach is known as an effective method to suppress unwanted noise $[1,2]$. As shown in Figure 1, a duct with a noise source (primary source), a secondary source, and an error microphone are considered to suppress the primary noise as much as necessary. A real-time algorithm which is embedded in a digital signal processor is applied for sound quality control as illustrated schematically in Figure 1 as well. The summation of the primary noise $d(n)$ and the secondary noise $y(n)$ at the error microphone is the error signal $e(n)$ as $e(n)=d(n)-y(n)$.

Assuming that there are $K$ reference signals, $M$ secondary loudspeakers, and $L$ error microphones, the practical form of the multichannel FxLMS update equation based on the steepest descent algorithm in the time domain without the sound quality factor can be given as $[1,2]$

$$
\mathbf{w}_{n+1}=\mathbf{w}_{n}-\alpha \widehat{\mathbf{X}}^{T}(n) \mathbf{e}(n),
$$

where $\mathbf{w}$ is the vector of filter coefficient, $\alpha$ is a convergence coefficient, and $\mathbf{e}(n)$ is the error signal vector.
$\widehat{\mathbf{X}}(n)$ is the filtered-reference signal matrix which can be expressed as

$$
\widehat{\mathbf{X}}(n)=\left[\begin{array}{cccc}
\widehat{\mathbf{x}}_{1}^{T}(n) & \widehat{\mathbf{x}}_{1}^{T}(n-1) & \cdots & \widehat{\mathbf{x}}_{1}^{T}(n-I+1) \\
\widehat{\mathbf{x}}_{2}^{T}(n) & \widehat{\mathbf{x}}_{2}^{T}(n-1) & \cdots & \widehat{\mathbf{x}}_{2}^{T}(n-I+1) \\
\vdots & \vdots & \ddots & \vdots \\
\widehat{\mathbf{x}}_{L}^{T}(n) & \widehat{\mathbf{x}}_{L}^{T}(n-1) & \cdots & \widehat{\mathbf{x}}_{L}^{T}(n-I+1)
\end{array}\right],
$$

where the filtered-reference signal vector can be written by

$$
\begin{aligned}
& \widehat{\mathbf{x}}_{\ell}(n) \\
& =\left[\begin{array}{lllllll}
\hat{x}_{\ell 11}(n) & \widehat{x}_{\ell 12}(n) & \cdots & \widehat{x}_{\ell 1 K}(n) & \widehat{x}_{\ell 21}(n) & \cdots & \widehat{x}_{\ell M K}(n)
\end{array}\right]^{T} .
\end{aligned}
$$

In (1), the practical update quantity in the multichannel system is given as $\partial J / \partial \mathbf{w}$, where $J$ is the cost function and is identical to the gradient of the instantaneous sum of squared outputs of the error signals with respect to the filter weights. Thus it can be presented as follows $[1,2]$ :

$$
\begin{aligned}
\frac{\partial J}{\partial \mathbf{w}(n)} & =\frac{\partial \mathbf{e}^{T}(n) \mathbf{e}(n)}{\partial \mathbf{w}(n)} \\
& =2\left[\widehat{\mathbf{X}}^{T}(n) \mathbf{X}(n) \mathbf{w}(n)+\widehat{\mathbf{X}}^{T}(n) \mathbf{d}(n)\right] \\
& =2 \widehat{\mathbf{X}}^{T}(n) \mathbf{e}(n) .
\end{aligned}
$$

In this control system, sound quality can be controlled using a digital equalizer [2]. The quality factor matrix $\mathbf{B}$, which consists of each quality factor $\beta_{i}$ to each secondary loudspeaker as presented in Figure 1, can be considered in the multichannel FxLMS algorithm and can allow actively controlling sound quality in a duct system. The output vector $\mathbf{u}(n)$ from the adaptive filter matrix $\mathbf{W}$ is designed to separate and to multiply with $\mathbf{I}-\mathbf{B}$ for the actual secondary path matrix $\mathbf{S}$ in one branch and $\mathbf{B}$ for the modelled one $\widehat{\mathbf{S}}$ in the other 
branch. This makes the error signal vector capable of being written by

$$
\mathbf{e}(n)=\mathbf{d}(n)-(\mathbf{I}-\mathbf{B}) \sum_{p=1}^{P-1} \mathbf{S}_{p} \mathbf{u}(n-p) .
$$

This error signal vector now indicates that the residual noise after control can be kept by the quality factor matrix $\mathbf{B}$ [5]. Thus, the practical update equation of the multichannel FxLMS algorithm for ASQC with the quality factor matrix B is given by

$$
\begin{aligned}
& \mathbf{w}_{n+1}=\mathbf{w}_{n}+\alpha \widehat{\mathbf{X}}^{T}(n)\left[\mathbf{e}(n)-\mathbf{B} \sum_{p=1}^{P-1} \widehat{\mathbf{S}}_{p} \mathbf{u}(n-p)\right] \\
&= \mathbf{w}_{n}+\alpha \widehat{\mathbf{X}}^{T}(n)\left[\left\{\mathbf{d}(n)-(\mathbf{I}-\mathbf{B}) \sum_{p=1}^{P-1} \mathbf{S}_{p} \mathbf{u}(n-p)\right\}\right. \\
&\left.-\mathbf{B} \sum_{p=1}^{P-1} \widehat{\mathbf{S}}_{p} \mathbf{u}(n-p)\right]=\mathbf{w}_{n}+\alpha \widehat{\mathbf{X}}^{T}(n) \mathbf{e}^{\prime}(n) .
\end{aligned}
$$

The new update equation in (6) operates automatically to minimize the signal vector $\mathbf{e}^{\prime}(n)$. So if the disturbance signal vector $\mathbf{d}(n) \approx \sum_{p=1}^{P-1} \mathbf{S}_{p} \mathbf{u}(n-p)$, then (5) can be expressed as

$$
\mathbf{e}(n) \approx \mathbf{B d}(n) .
$$

Equation (7) represents the quality factor matrix governing the residual noise level at the error microphones positions in the duct. The separation of the signal $y_{i}$ introduces four different cases of results as follows: (1) if $\beta_{i}=0$, it acts as a normal ANC system which is on (this is equivalent to (1)) and makes $e_{i}(n) \approx 0$, (2) if $\beta_{i}=1$, it acts as the ANC system which is off and makes $e_{i}(n) \approx d_{i}(n)$, (3) if $0<\beta_{i}<1$, it acts to control the noise to a certain level dependent upon the value $\beta_{\mathrm{i}}$ and makes $e_{i}(n) \approx \beta_{i} d_{i}(n)$, and (4) if $\beta_{i}>1$, it acts to enhance the noise level and makes $e_{i}(n)>d_{i}(n)$.

\section{Implementation and Experiment}

3.1. Experimental Set-Up and Primary Path. The experimental set-up for the real-time narrowband active sound quality control consists of an acrylic duct (length $=1800 \mathrm{~mm}$ ), a primary loudspeaker at the left end, two secondary sources (control loudspeakers), an error microphone $\left(1 / 2^{\prime \prime} \mathrm{PCB}\right.$ 377B0E) at the right end, a power amplifier (B\&K 2716C), a PCB signal conditioner, and low-pass filters. In addition, it involves the real-time control unit of a dSPACE 1104 for the implementation of control algorithm. So there are 1 reference signal $(K=1), 2$ secondary loudspeakers $(M=2)$, and 1 error microphone $(L=1)$ in this control system.

The sampling frequency was $f_{s}=6.000 \mathrm{kHz}$ and the cut-off frequencies of the antialiasing low-pass filters before the two ADCs (for the reference signal and the error signal) and the reconstruction low-pass filter after the DAC (for the control signal) were both $500 \mathrm{~Hz}$. The control signal vector $\mathbf{u}(n)$, which was generated in the control algorithm, was transferred to the control loudspeakers through the DAC. The physical acoustic path lengths between the secondary sources and the error microphone are $1140 \mathrm{~mm}$ for the source 1 and $600 \mathrm{~mm}$ for source 2 , respectively.

In a duct system, fan noise is the most dominant one in general. Thus a narrowband signal is highly likely as the input. Other different signals pure tones or broadband signals can be the input. Control against pure tones is very easy but the usefulness in practical applications is very low. Control against broadband signals is useful but the signals require very long control filters for successful control. However, many practical applications such as fan, motors, engines, and other rotating devices generate nonstationary narrowband signals. The narrowband control based on the adaptive notch filter needs two filter coefficients per order, and this allows less control filter lengths. By the way, this study investigates the narrowband signal which consists of three different orders.

Thus, a narrowband signal with three components of $\mathrm{C} 1$ (mode 1), C1.5 (mode 2), and C2 (mode 3 ) is considered for the primary disturbance noise as shown in Figure 2(a).

The spectrogram of the disturbance signal in Figure 2(a) indicates that three components are involved as described previously and it stays stationarily for the first and the last 2 seconds, respectively, but sweeps nonstationarily for 5 seconds between the two stationary signals. During the sweep, $\mathrm{C} 1, \mathrm{C} 1.5$, and $\mathrm{C} 2$ order signals vary from $100 \mathrm{~Hz}$ to $200 \mathrm{~Hz}, 150 \mathrm{~Hz}$ to $300 \mathrm{~Hz}$, and $200 \mathrm{~Hz}$ to $400 \mathrm{~Hz}$, respectively.

In Figure 2(b), the block diagram of the multichannel narrowband FxLMS which was implemented in DSPACE 1104 is displayed. The block diagram shows adaptive notch filters to cancel actively the narrowband disturbances. The block diagram in Figure 2(b) is the extended version of Figure 1 to generate proper control signals to operate the two secondary loudspeakers. Each dashed rectangle in Figure 2(b) represents an adaptive notch filter against one of the three orders to drive one of the two loudspeakers.

3.2. Secondary Path Modelling. The plants of the active control system which is known as secondary paths between each control loudspeaker, LS1 or LS2, and the error microphone were measured with the dSPACE 1104 as plotted in Figure 1. Both the plant models $\widehat{S}_{1}(z)$ and $\widehat{S}_{2}(z)$ were obtained using the offline identification method from the measured data. As it can be seen from Figure 3, the frequency response function (FRF) and the impulse response functions (IRF) are plotted.

The FIR filters $\widehat{s}_{1}(n)$ and $\widehat{s}_{2}(n)$ were implemented for the plant models in the control algorithm as shown in Figure 2(b). The lengths of the FIR filters were 50 samples, respectively, although the IRFs in Figure 3 show 200 samples. The length of $\widehat{s}_{1}(n)$ depends upon the performance of the control processor for the real-time control and the complexity of the algorithm used.

The lengths of the secondary path models in FIR filters were chosen after analyzing the mean square of the error signal which is the difference of the outputs between the actual secondary path and the secondary path model. The analysis showed that the length of 50 samples is reasonable to choose as it offers nice control performance in terms of the 


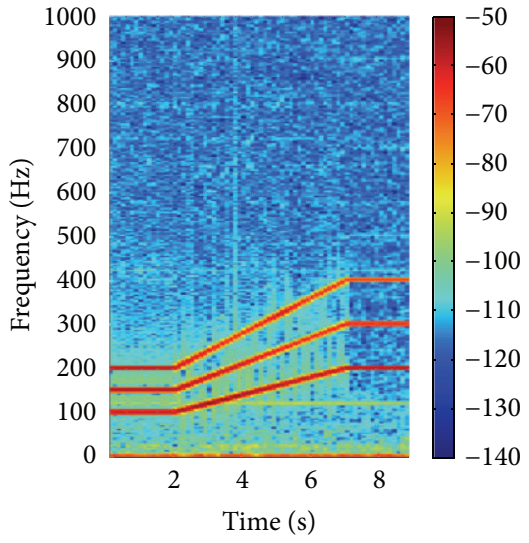

(a)

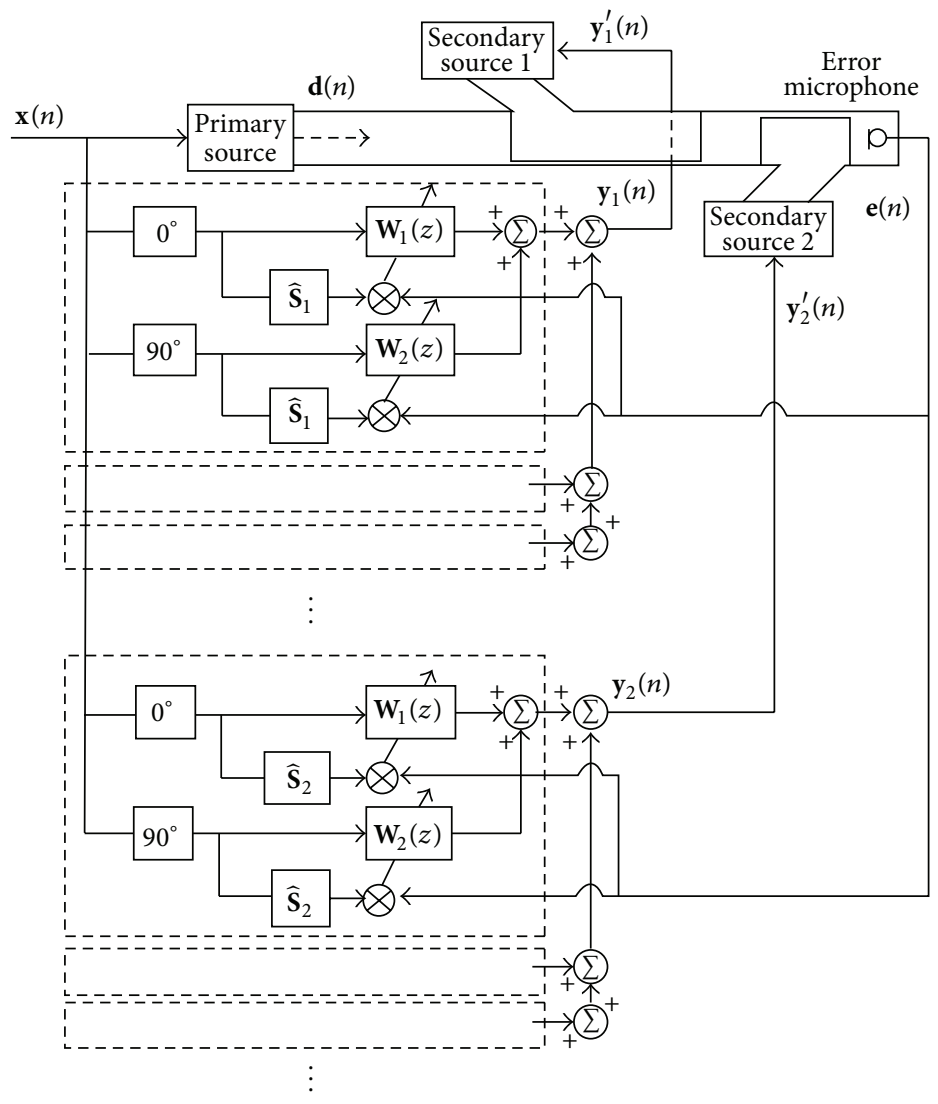

(b)

FIGURE 2: Disturbance and the control algorithm. (a) Spectrogram of the disturbance signal. (b) Implementation of the multichannel FxLMS algorithm for narrowband noise control.

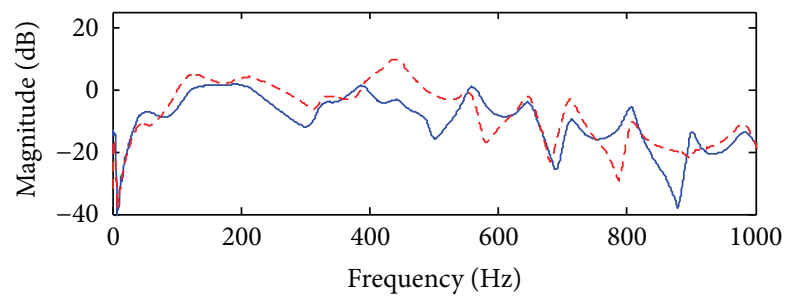

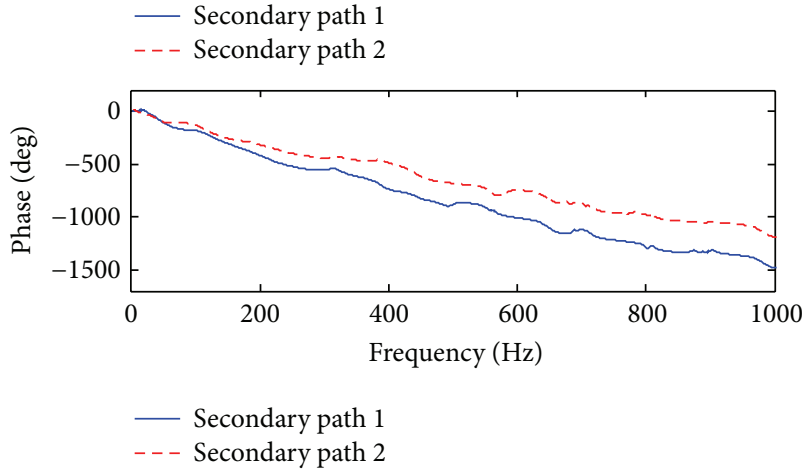

(a)
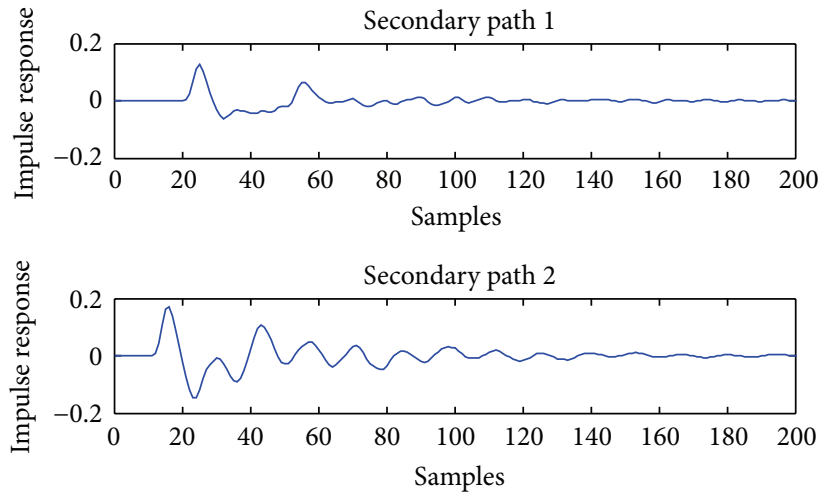

(b)

FIGURE 3: Modelling of the two secondary paths. (a) Frequency response function (b) impulse response function of the modelled plants $\widehat{S}_{1}(z)$ and $\widehat{S}_{2}(z)$. 


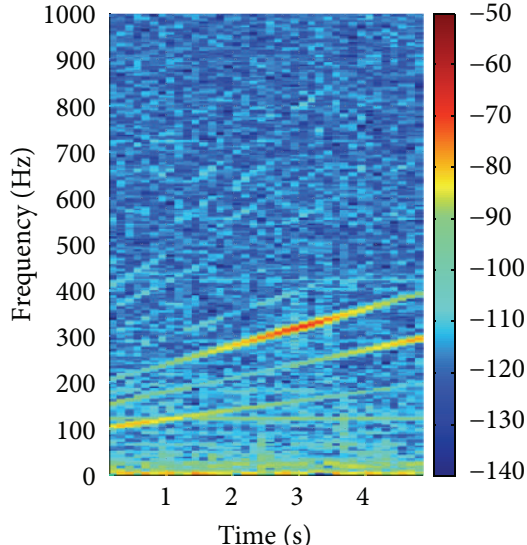

(a)

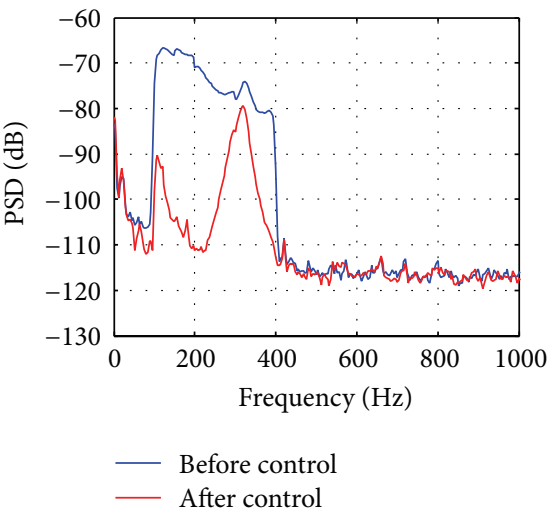

(d)

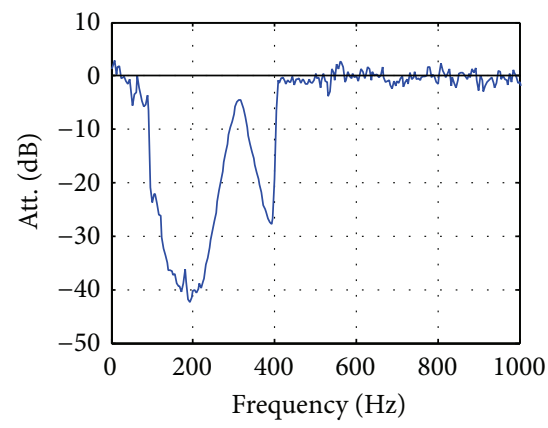

(g)

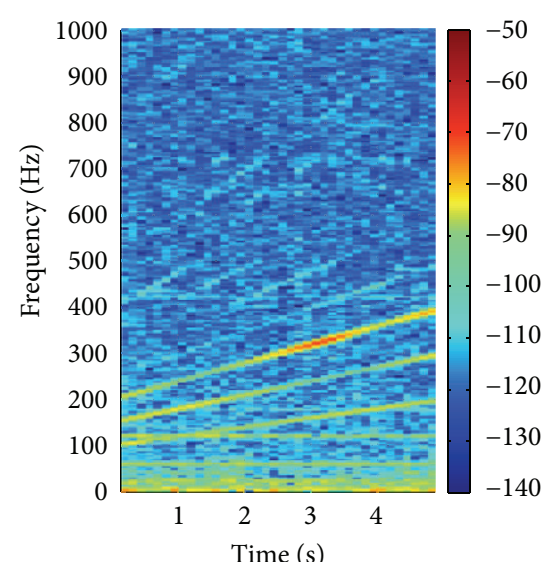

(b)

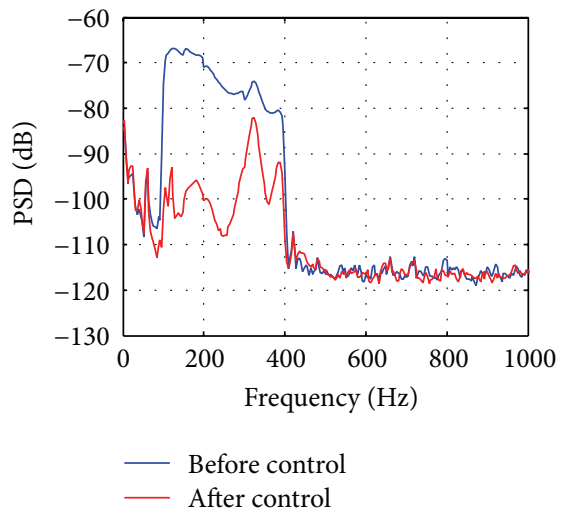

(e)

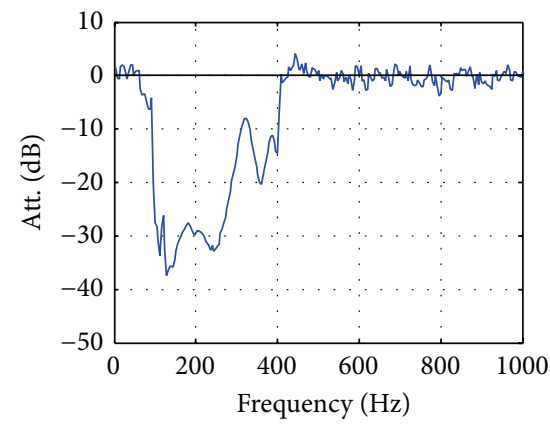

(h)

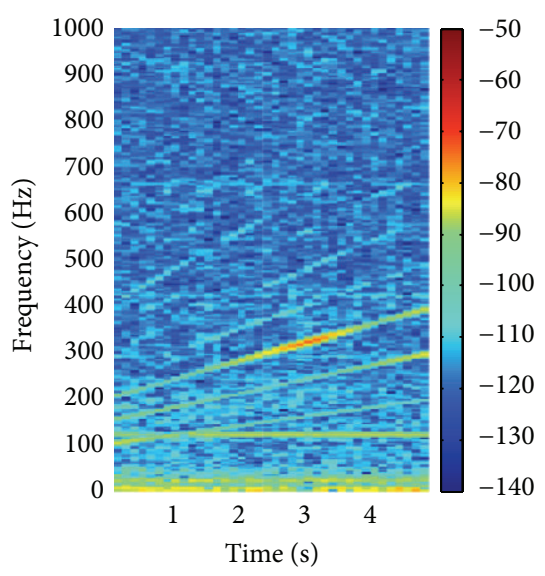

(c)

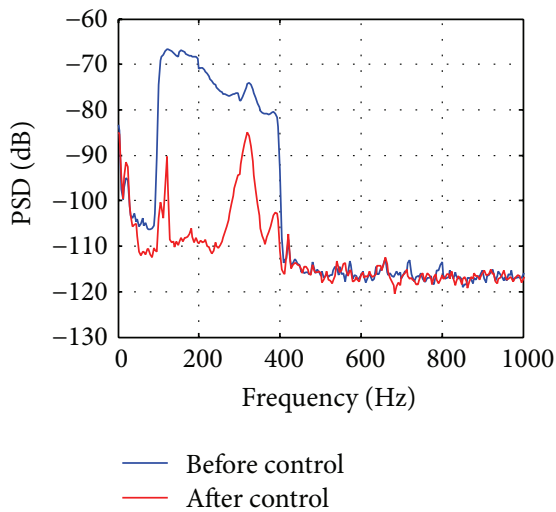

(f)

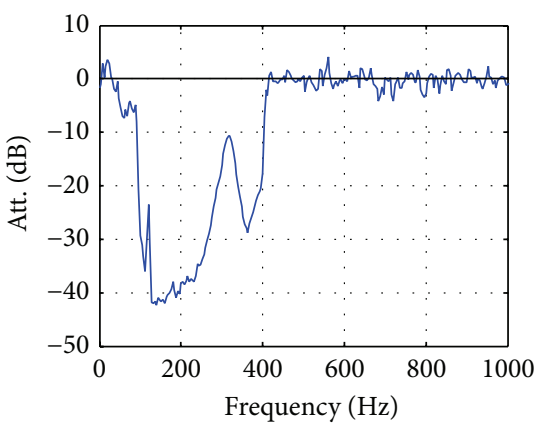

(i)

FIGURE 4: Real-time narrowband control results without the quality factor. ((a), (b), (c)) Spectrograms after control when LS1 only, LS2 only, and LS1 + LS2 are operated, respectively. ((d), (e), (f)) Comparison of error spectra between "before control" and "after control." ((g), (h), (i)) Comparison of attenuations.

MSE (mean square error) and real-time computation time. The longer filter length gives the further reduction in control but it requires further real-time processing capability.

\section{Results and Discussions}

In this experiment, either LS1 or LS2 or both (LS1 + LS2) the two secondary loudspeakers were used for the control.
Also the control results present without $(\beta=0)$ and with the quality factor $(0 \leq \beta \leq 1)$.

4.1. Active Control Results without the Quality Factor $(\beta=0)$. When the quality factor was not applied $(\beta=0)$ to the control implementation, the real-time control experiment results show the full performance with the largest attenuations as shown in Figure 4. Figures 4(a), 4(d), and 4(g) indicate the spectrogram of the error signals after control, comparison of 
the error spectra $S_{e e}(f)$ between before and after control, and the attenuation $\operatorname{Att}(f)$, respectively, when only LS1 is used. The attenuation is defined as follows:

$$
\begin{aligned}
\operatorname{Att}(f) & =10 \log _{10}\left[\frac{S_{e e, \text { after }}(f)}{S_{e e, \text { before }}(f)}\right] \\
& =10 \log _{10}\left[\frac{S_{e e, \text { after }}(f)}{S_{d d}(f)}\right],
\end{aligned}
$$

where the subscripts after and before represent "after control" and "before control," respectively, and $S_{d d}(f)$ is the disturbance spectrum.

When only LS1 is used, the spectrogram of the error signals after control, compared with Figure 2(a), shows that three sweeping components $(\mathrm{C} 1, \mathrm{C} 1.5$, and $\mathrm{C} 2)$ are attenuated very well. The average attenuation level over the frequency range of $100-400 \mathrm{~Hz}$ in this case is about $25.62 \mathrm{~dB}$ as summarized in Table 1.

Figures 4(b), 4(e), and 4(h) show that the average attenuation level of the error signals over the same frequency range is about $24.09 \mathrm{~dB}$ as only LS2 is used. Also Figures 4(c), 4(f), and 4(i) demonstrate that the average attenuation level is about $29.74 \mathrm{~dB}$ when both LS1 and LS2 are used.

It is noted that the case with both LS1 and LS2 shows the best performance compared with the other two cases. It is eminent from the results in Figure 4 that the frequency region especially around $320 \mathrm{~Hz}$ (C3) is not controlled effectively. This is caused by the fact that the eigenvalue spread is inherently wider than other regions in this duct control system.

The less controlled peak around $300 \mathrm{~Hz}$ in Figure 4 is caused by the sudden phase change in the secondary path around that frequency as plotted in Figure 3(a), which is determined by the physical dimension of the duct and the physical and electrical properties between the secondary loudspeakers and the error microphone. In active control, the sudden phase change at a certain frequency especially limits the convergence coefficient value in order to maintain the stability of the control system. So the sudden phase change determines the control performance. Once the convergence coefficient value increases, the peak around that frequency can be increased abruptly and threatens the stability. Thus even if the duct dimension cannot be redesigned, one can reduce the amount of the phase change at a certain frequency by modifying the physical and electrical properties of the secondary paths. Then the change can lead to better control performance.

From Table 1, the result differences between LS1 only and LS2 are observed although they are not big. This is mainly caused by the differences in terms of the physical and electrical properties between the two secondary paths. The convergence coefficient values are different slightly in the two paths and bring about the result difference.

In addition, although it might look so natural this justifies the fact that the use of more control loudspeakers can introduce better attenuation in active noise control provided that the complexity of the algorithm is not beyond the
TABLE 1: Average attenuation in $\mathrm{dB}$ of the error signals without/with

\begin{tabular}{|c|c|c|c|}
\hline & LS1 only (dB) & LS2 only $(\mathrm{dB})$ & $\mathrm{LS} 1+\mathrm{LS} 2(\mathrm{~dB})$ \\
\hline $\mathrm{C} 1(\beta=0.0)$ & 5.28 & 5.97 & - \\
\hline $\mathrm{C} 1.5(\beta=0.0)$ & 1.40 & 1.22 & - \\
\hline $\mathrm{C} 2(\beta=0.0)$ & 6.03 & 5.82 & - \\
\hline $\begin{array}{l}\mathrm{C} 1, \mathrm{C} 1.5, \mathrm{C} 2(\beta=1.0) \\
\text { Control off }\end{array}$ & 0.00 & 0.00 & 0.00 \\
\hline $\begin{array}{l}\mathrm{C} 1, \mathrm{C} 1.5, \mathrm{C} 2(\beta=0.5) \\
\text { Quality control on }\end{array}$ & - & - & 5.84 \\
\hline $\begin{array}{l}\mathrm{C} 1, \mathrm{C} 1.5, \mathrm{C} 2(\beta=0.2) \\
\text { Quality control on }\end{array}$ & - & - & 13.71 \\
\hline $\begin{array}{l}\mathrm{C} 1, \mathrm{C} 1.5, \mathrm{C} 2(\beta=0.0) \\
\text { Control on }(\text { full })\end{array}$ & 25.62 & 24.09 & 29.74 \\
\hline
\end{tabular}
the quality factor.

computation power of the real-time processor and the control is stable.

4.2. Active Control Results for Each Component without the Quality Factor $(\beta=0)$. Again if the quality factor was not applied $(\beta=0)$ to the narrowband control implementation, the spectrograms of the error signals after control on each component by using either LS1 only or LS2 only are plotted in Figure 5.

Figures 5(a), 5(b), and 5(c) show that C1, C1.5, and C2 components are cancelled out by using LS1 only. The noise at about $320 \mathrm{~Hz}$ in C2 is still not suppressed even if the control is dedicated only to one component (C2) by LS1. The average attenuation levels in this case are summarized in Table 1.

In Figures 5(d), 5(e), and 5(f), the spectrograms of the error signals after control are quite similar to Figures 5(a), 5(b), and 5(c). It is noted that the average attenuation at C1.5 with LS2 is a bit better than LS1 from Figures 5(b) and 5(e), although the average attenuation level in Table 1 shows differently. Because the $\mathrm{C} 1.5$ which extends from $150 \mathrm{~Hz}$ to $300 \mathrm{~Hz}$ overlaps with $\mathrm{C} 1$ at $150-200 \mathrm{~Hz}$ and C2 at $200-300 \mathrm{~Hz}$, the average attenuation levels for $\mathrm{C} 1.5$ cannot represent actual results in this case.

4.3. Active Control Results with the Quality Factor $(0 \leq \beta \leq$ $1)$. In this section, three different quality factors of $\beta=0$, $\beta=0.2$, and $\beta=0.5$ were applied to the actual control implementation; the real-time control experiment results in terms of their attenuations when both LS1 and LS2 are used are shown in Figure 6. As Figures 6(a), 6(b), and 6(c) indicate the attenuation at $\beta=0$ (full control), $\beta=0.2$, and $\beta=0.5$, respectively, the largest attenuation was observed at $\beta=0$.

The control results including the error spectrum $S_{e e}(f)$ and the attenuation $\operatorname{Att}(f)$ in the frequency domain are presented in Figures 7(a) and 7(b) with 4 different $\beta$ of 1.0 (before control, thin lines), 0.5 (quality control, dashed lines), 0.2 (quality control, thick lines), and 0.0 (full control, thickest lines). 


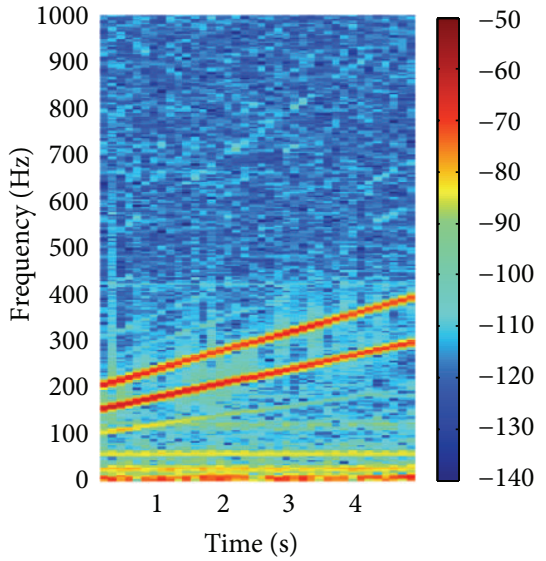

(a)

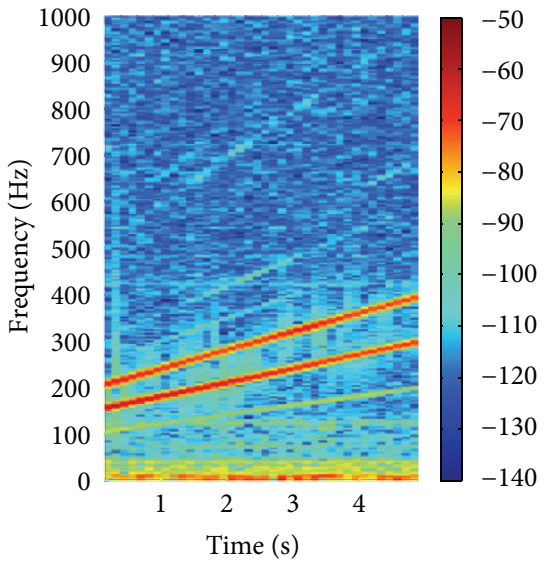

(d)

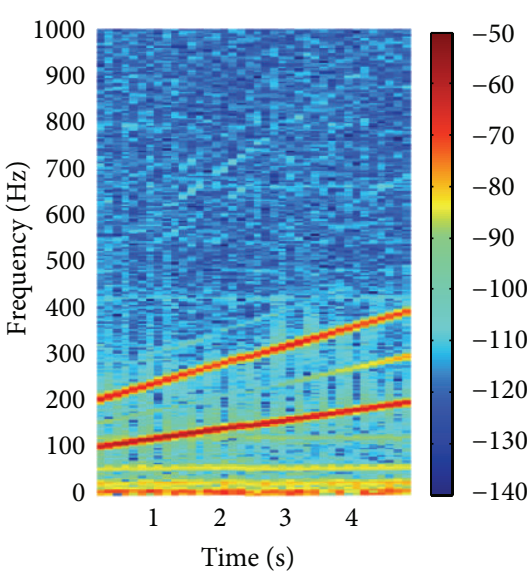

(b)

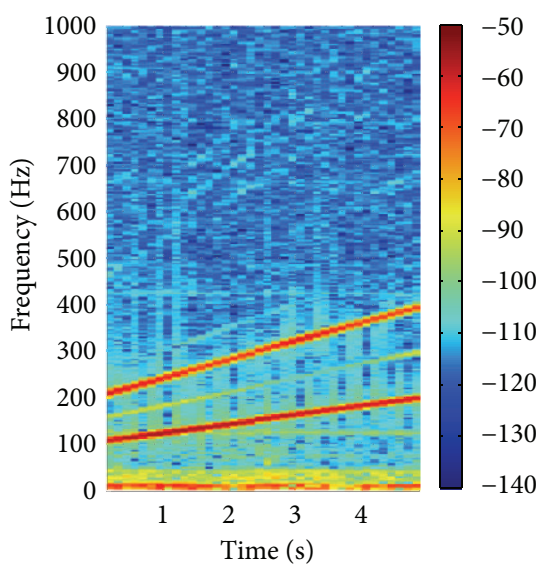

(e)

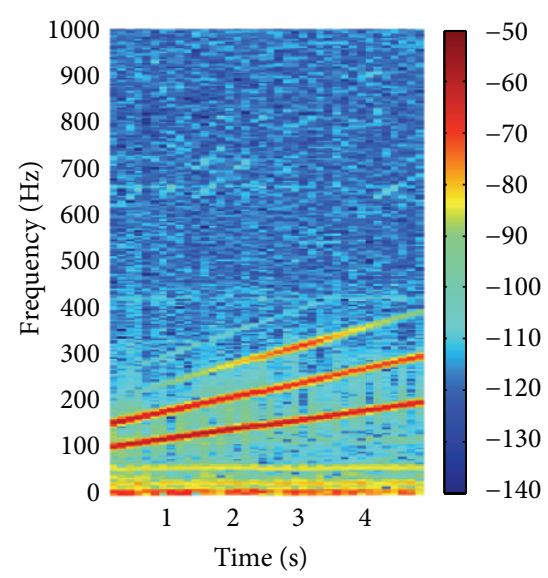

(c)

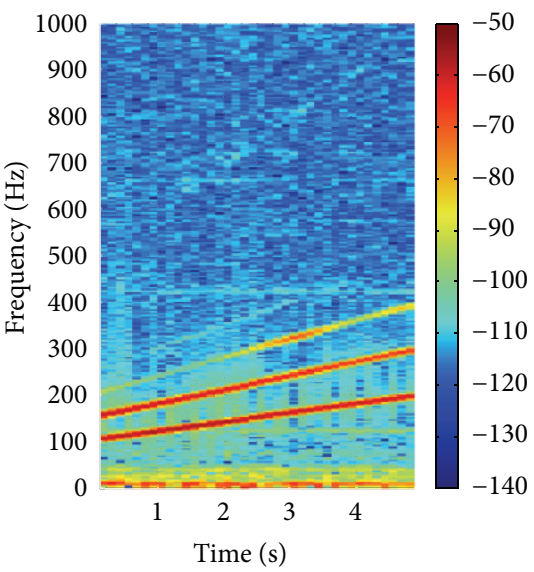

(f)

FIGURE 5: Spectrograms of the error signals from real-time narrowband control results for each component without the quality factor. (a) C1 control with LS1. (b) C1.5 control with LS1. (c) C2 control with LS1. (d) C1 control with LS2. (e) C1.5 control with LS2. (f) C2 control with LS2.

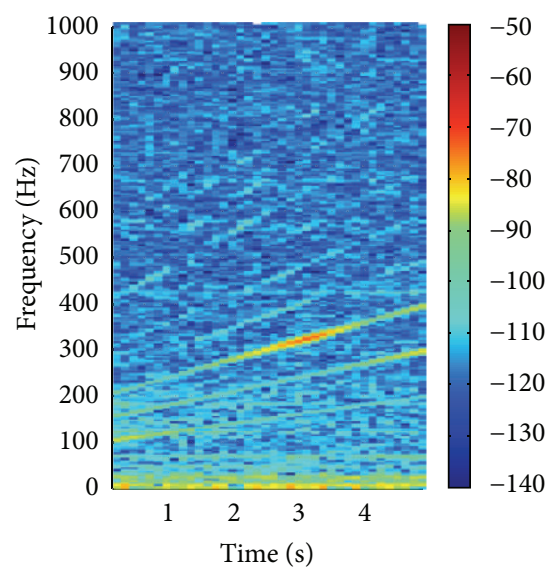

(a)

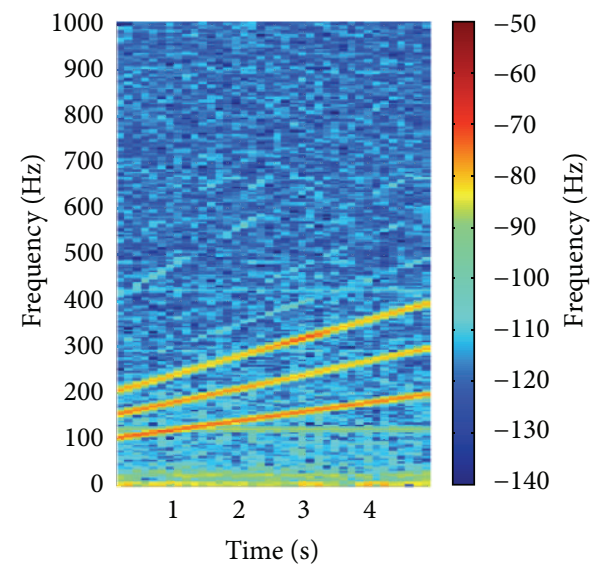

(b)

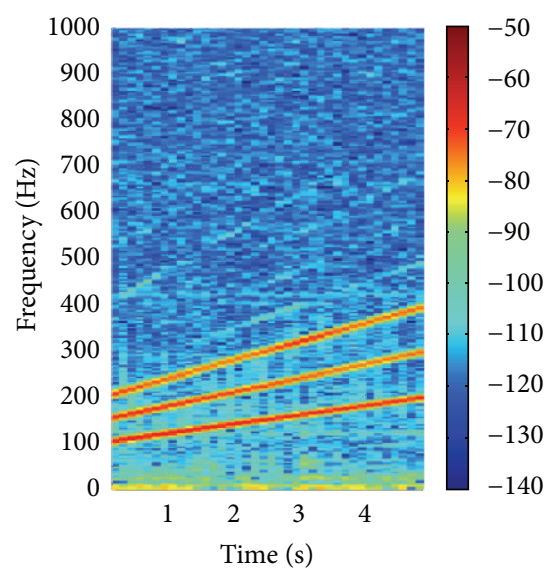

(c)

FIGURE 6: Spectrograms of the error signals from real-time narrowband control results with the quality factor when both LS1 and LS2 are operated. (a) $\beta=0$. (b) $\beta=0.2$. (c) $\beta=0.5$. 


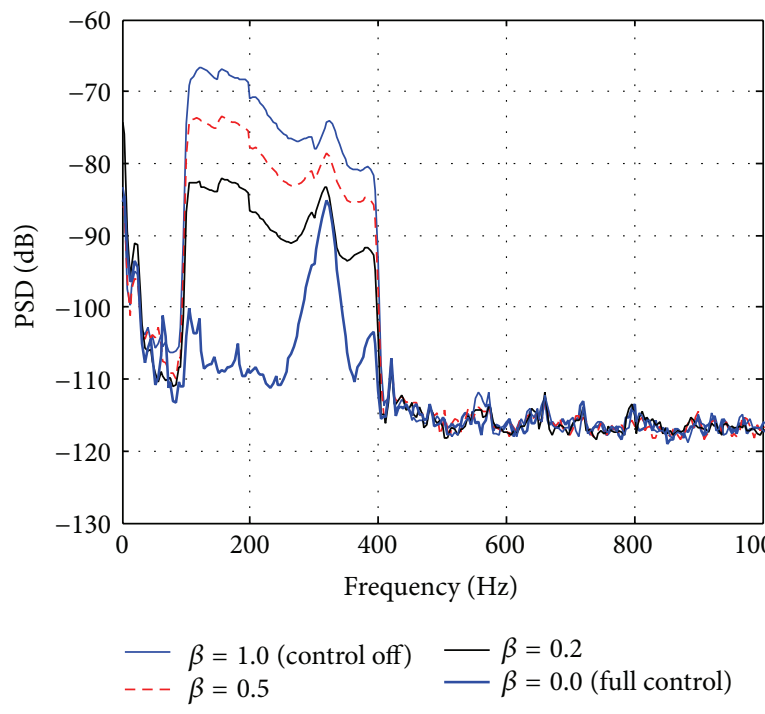

(a)

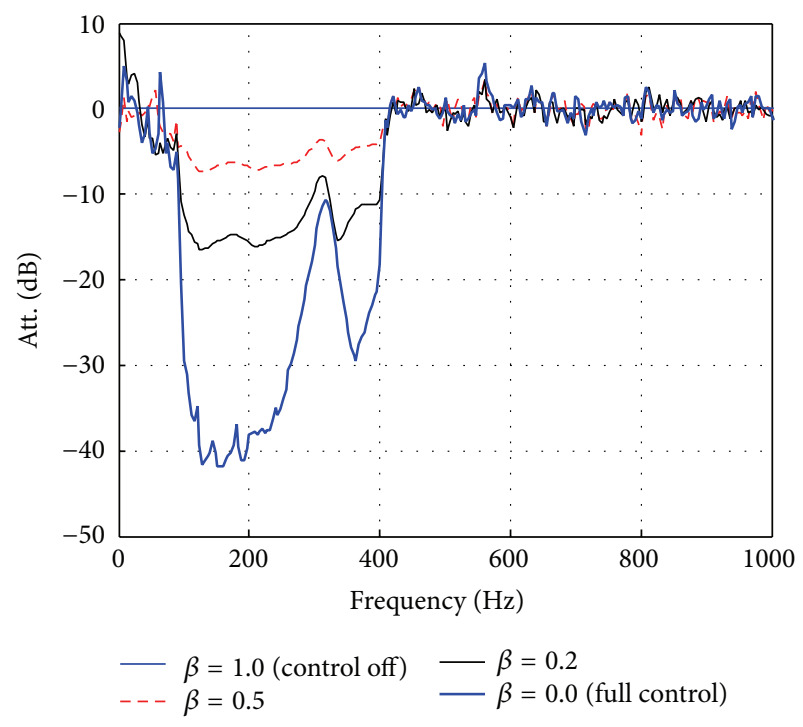

(b)

FIGURE 7: Comparison of control performances in terms of error spectra and attenuation between before control ( $\beta=1.0$ : thin lines) and after control ( $\beta=0.5$ : dashed lines, $\beta=0.2$ : thick lines, and $\beta=0.0$, thickest lines) against narrowband noise. (a) Error spectra. (b) Attenuations.

Figure 7 shows the effect of the sound quality factor variation dramatically in both the error spectra and the attenuation. As summarized in Table 1 , the average attenuations over the frequency range of $100 \mathrm{~Hz}-400 \mathrm{~Hz}$ for $\beta=0.0$, $\beta=0.2, \beta=0.5$, and $\beta=1.0$ are $29.74 \mathrm{~dB}, 13.71 \mathrm{~dB}, 5.84 \mathrm{~dB}$, and $0.00 \mathrm{~dB}$, respectively.

It is obvious that the lower $\beta$ cancels the more primary noise as defined in the theory although the attenuation levels are not exactly and inversely proportional to the quality factor $\beta$. It is also observed that there are no spillover phenomena after control beyond the frequency of $400 \mathrm{~Hz}$ in the low-pass filters and this indicates that this approach operates with high stability and very nice performance over a wide frequency range.

The control results shown in Figures 6 and 7 demonstrate that any specific acoustic modes can be suppressed as much as it is necessary by using the quality factor $\beta$. This can be extended to even more complicated sound quality control systems such as vehicles or other products.

By the way, the optimal quality factor can be calculated when a target profile is given before control in this approach. The quality factor can be adapted automatically during control when the target profile over frequency is predefined before control. For the implementation of those systems, it is necessary to insert the command input (target profile) signal into the block diagram in Figure 1; then the difference between the command input and the error signal is feedback to the LMS algorithm.

As an ASQC system requires controlling the primary noise to a certain target profile over frequency, it is expected that this approach could provide a practical solution.

In addition, if the primary input signal is broadband, the quality factor will work equally over the frequency range. This will lead to attenuation of the noise level but the amount of the attenuation might be less than that for the narrowband input signal.

\section{Conclusions}

This study presents active sound quality control of some acoustic modes $(\mathrm{C} 1, \mathrm{C} 1.5$, and $\mathrm{C} 2$ components) in a 1dimensional duct by means of the quality factor $\beta$ manipulation, which was implemented in the real-time multichannel FxLMS algorithm based on biologically inspired learning. Control results show that the algorithm with or without the quality factor provided stable and excellent responses in experiments compared to before control. As either or both the two control loudspeakers in the duct can be used, the three components were controlled at the same time or separately with large attenuations.

For the individual control with either LS1 or LS2, the average attenuations are quite similar with or without the quality factor. LS1 especially showed $25.62 \mathrm{~dB}$ reduction in overall when beta $=0$, but LS2 gave $24.09 \mathrm{~dB}$ reduction.

The quality factor value influences tremendously control results; as the values decrease with beta $=1.0,0.5,0.2$, and 0.0 , the attenuation levels increase to $0.00,5.84,13.71$, and $29.74 \mathrm{~dB}$ when LS1 and LS2 are used.

It is obvious that the lower $\beta$ cancels the more primary noise as defined in the theory although the attenuation levels are not exactly and inversely proportional to the quality factor. The multichannel sound quality algorithm worked with nice stability and performance against a narrowband noise. The results in this study can be used for practical active sound quality control systems.

For the future, a target profile based control will be investigated to extend this study for actual application 
to a large duct system. The new control scheme will contain the command input (target profile over frequency) and the input will be compared with the error signal to drive the adaptive FxLMS algorithm. The quality factor will be automatically updated at each sample to meet the target profile.

\section{Conflict of Interests}

The authors declare that there is no conflict of interests regarding the publication of this paper.

\section{Acknowledgment}

This work was supported by the Incheon National University Research Grant in 2012.

\section{References}

[1] Y. Kajikawa, W. S. Gan, and S. M. Kuo, "Recent advances on active noise control: open issues and innovative applications," APSIPA Transactions on Signal and Information Processing, vol. 1, article e3, 21 pages, 2012.

[2] L. V. Wang, W. S. Gan, A. W. Khong, and S. M. Kuo, "Convergence analysis of narrowband feedback active noise control system with imperfect secondary-path estimation," IEEE Transactions on Audio, Speech, and Language Processing, vol. 21, no. 11, pp. 2403-2411, 2013.

[3] Y.-S. Lee and S. J. Elliott, "Active position control of a flexible smart beam using internal model control," Journal of Sound and Vibration, vol. 242, no. 5, pp. 767-791, 2001.

[4] D. Bismor, "LMS algorithm step size adjustment for fast convergence," Archives of Acoustics, vol. 37, no. 1, pp. 31-40, 2012.

[5] S. J. Elliott, Signal Processing for Active Control, Academic Press, 2001.

[6] S. O. Haykin, Adaptive Filter Theory, Prentice-Hall, 5th edition, 2013.

[7] J. C. Burgess, "Active adaptive sound control in a duct: a computer simulation," The Journal of the Acoustical Society of America, vol. 70, no. 3, pp. 715-725, 1981.

[8] K. Lee, W. S. Gan, and S. M. Kuo, Subband Adaptive Filtering: Theory and Implementation, Wiley, 2009. 


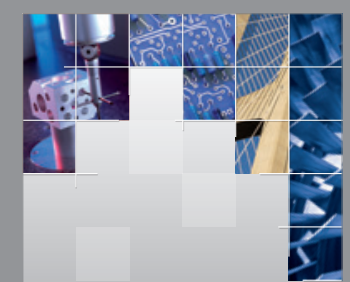

\section{Enfincering}
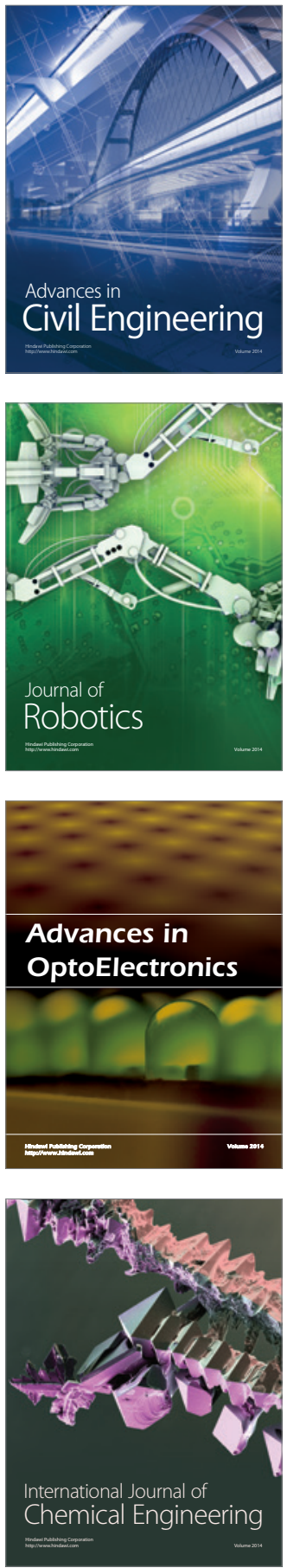

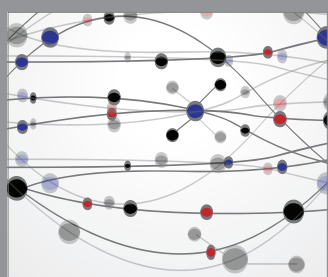

The Scientific World Journal

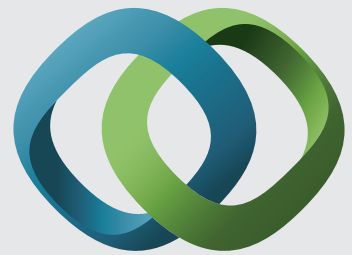

\section{Hindawi}

Submit your manuscripts at

http://www.hindawi.com
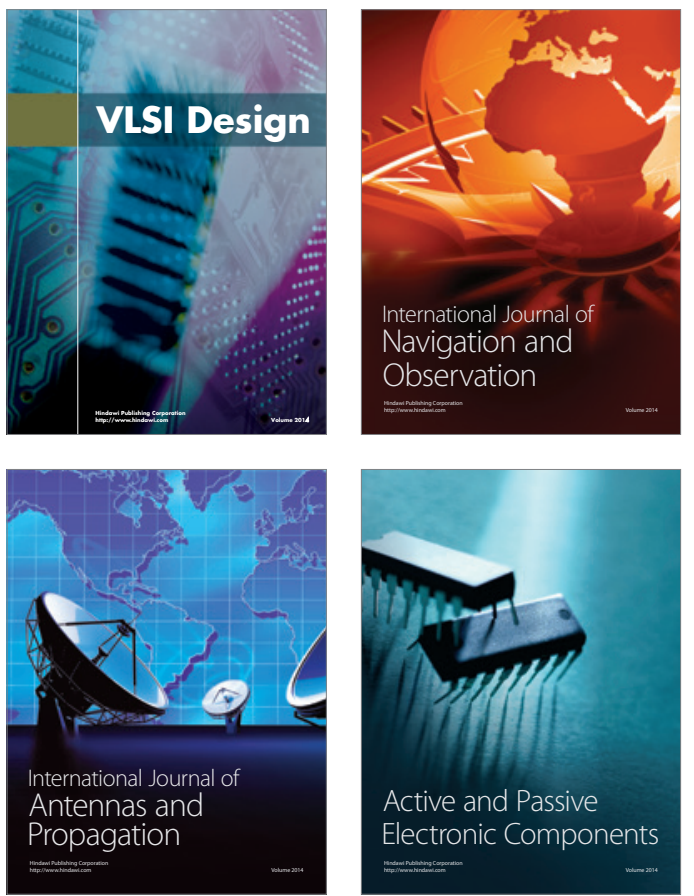
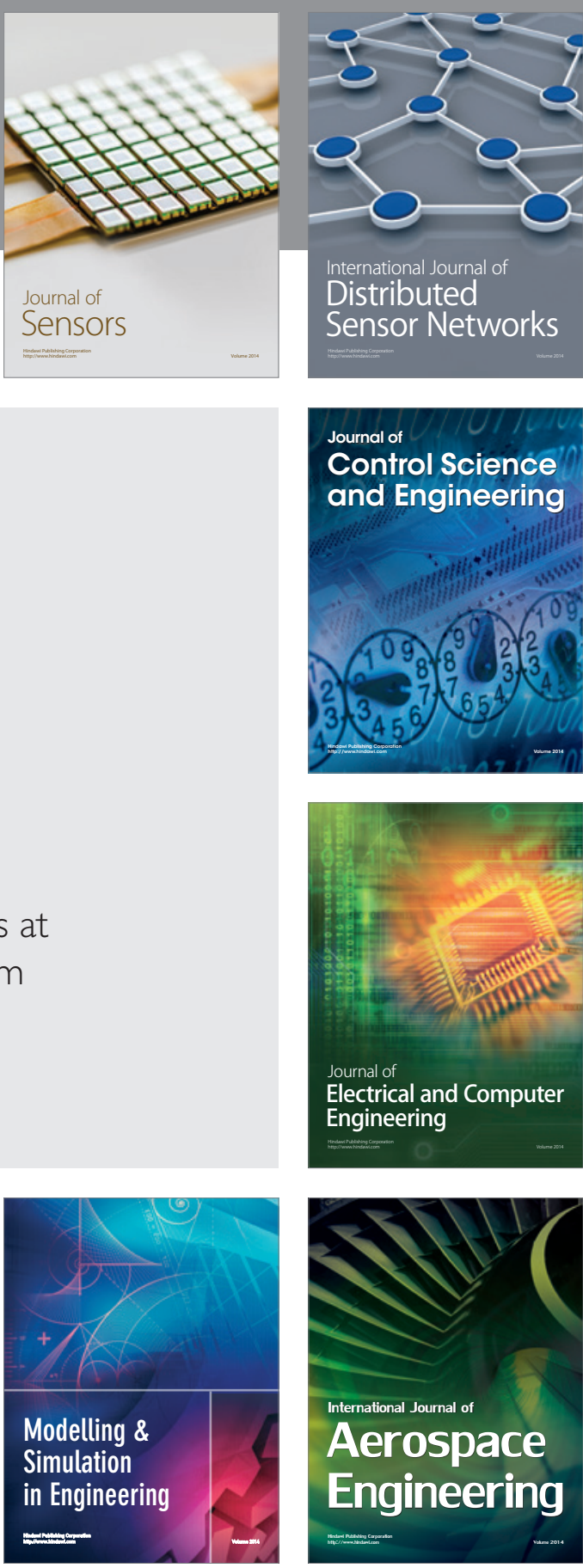

International Journal of

Distributed

Sensor Networks

Journal of

Control Science

and Engineering
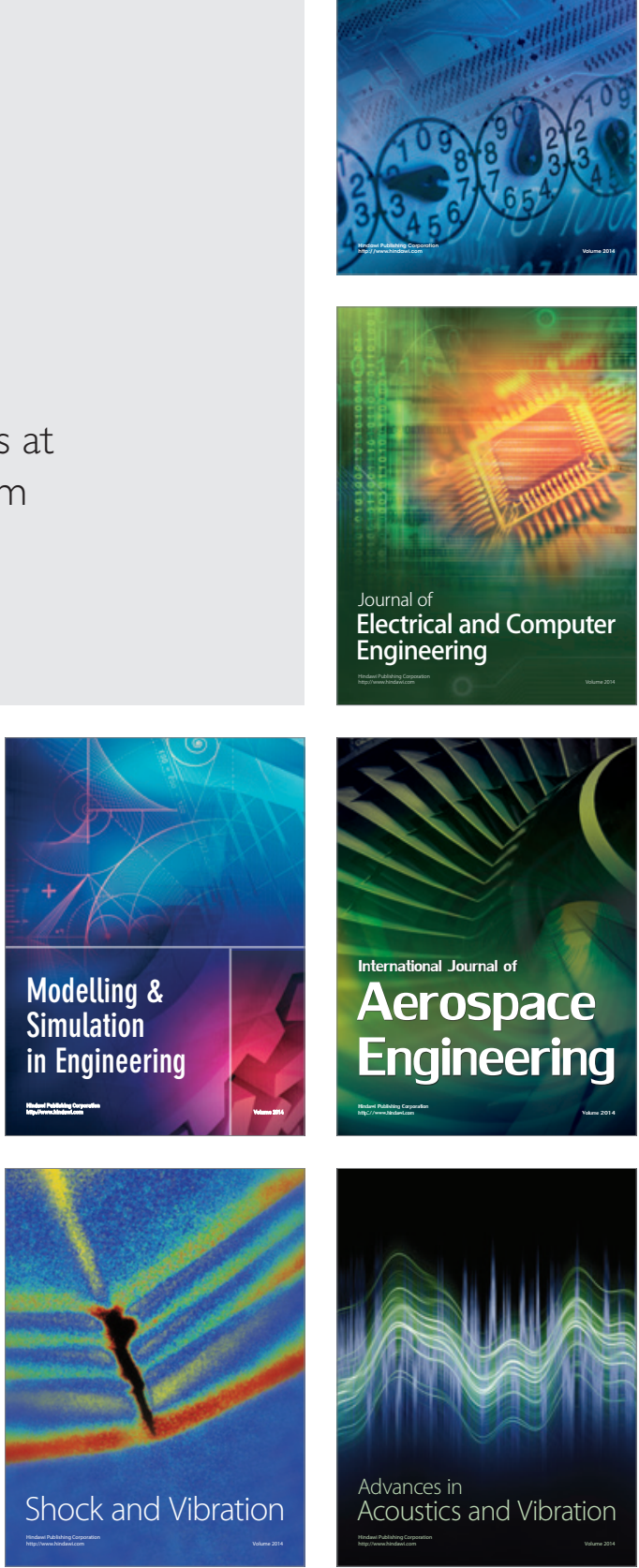